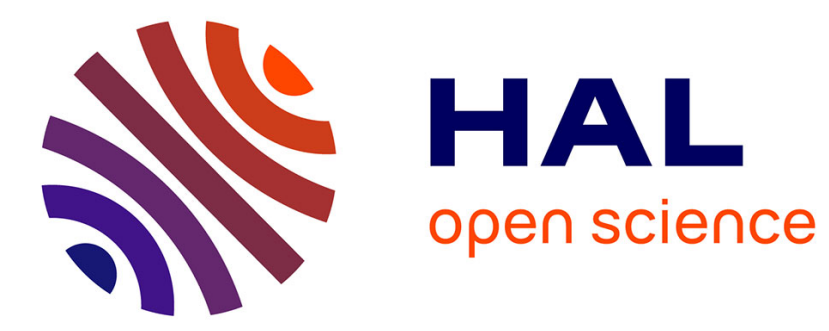

\title{
Stochastic Decomposition into Low Rank and Sparse Tensor for Robust Background Subtraction
}

\author{
Sajid Javed, Thierry Bouwmans, Soon Ki Jung
}

\section{To cite this version:}

Sajid Javed, Thierry Bouwmans, Soon Ki Jung. Stochastic Decomposition into Low Rank and Sparse Tensor for Robust Background Subtraction. 6th International Conference on Imaging for Crime Prevention and Detection (ICDP 2015), Jul 2015, Londres, United Kingdom. 10.1049/ic.2015.0105 . hal-01374218

\section{HAL Id: hal-01374218 \\ https://hal.science/hal-01374218}

Submitted on 30 Sep 2016

HAL is a multi-disciplinary open access archive for the deposit and dissemination of scientific research documents, whether they are published or not. The documents may come from teaching and research institutions in France or abroad, or from public or private research centers.
L'archive ouverte pluridisciplinaire HAL, est destinée au dépôt et à la diffusion de documents scientifiques de niveau recherche, publiés ou non, émanant des établissements d'enseignement et de recherche français ou étrangers, des laboratoires publics ou privés. 


\title{
Stochastic Decomposition into Low Rank and Sparse Tensor for Robust Background Subtraction
}

\author{
Sajid Javed $^{1}$, Thierry Bouwmans ${ }^{2}$, and Soon Ki Jung ${ }^{1}$ \\ ${ }^{1}$ School of Computer Science and Engineering, Kyungpook National University \\ 80 Daehakro, Buk-gu, Daegu, Republic of Korea \\ sajid@vr.knu.ac.kr, skjung@knu.ac.kr \\ ${ }^{2}$ Laboratoire MIA (Mathematiques, Image et Applications)- \\ Université de La Rochelle, 1700, France \\ thierry.bouwmans@univ-lr.fr
}

Keywords: Tensor decomposition, Low-rank tensor, Stochastic optimization, Background/Foreground Separation.

\begin{abstract}
Background subtraction (BS) is a very important task for various computer vision applications. Higher-Order Robust Principal Component Analysis (HORPCA) based robust tensor recovery or decomposition provides a very nice potential for $\mathrm{BS}$. The BG sequence is then modeled by underlying lowdimensional subspace called low-rank while the sparse tensor constitutes the foreground (FG) mask. However, traditional tensor based decomposition methods are sensitive to outliers and due to the batch optimization methods, high dimensional data should be processed. As a result, huge memory usage and computational issues arise in earlier approaches which are not desirable for real-time systems. In order to tackle these challenges, we apply the idea of stochastic optimization on tensor for robust low-rank and sparse error separation. Only one sample per time instance is processed from each unfolding matrices of tensor in our scheme to separate the low-rank and sparse component and update the low dimensional basis when a new sample is revealed. This iterative multi-dimensional tensor data optimization scheme for decomposition is independent of the number of samples and hence it reduces the memory and computational complexities. Experimental evaluations on both synthetic and real-world datasets demonstrate the robustness and comparative performance of our approach as compared to its batch counterpart without scarificing the online processing.
\end{abstract}

\section{Introduction}

Background Subtraction (BS) is a very important task in video analysis. This low level task consists of accurate and efficient isolation of moving objects called "Foreground" (FG) from the static information called "Background" (BG) scene. The neat and clean BG model is then further used for high level computer vision and image processing tasks such as video compression, privacy, inpainting, augmented reality, computational photography, etc. [1] [10]. However, the task becomes more complex when the data size grows since the real-world scenario requires larger data to be processed in a more efficient way.

Many algorithms have been proposed for BG/FG segmentation and several implementations are available in the $\mathrm{BGS}^{1}$ library. Among them subspace learning model such as HigherOrder Robust Principal Component Analysis (HORPCA) based tensor decomposition provides a very nice framework for moving object detection. Tensor based decomposition methods [7] [19] also known as HORPCA have been recently proposed for exact low-rank recovery which exploits information using multidimensional $\mathrm{N}$-arrays and provides an equivalent and more efficient framework than traditional RPCA-based approach [5]. LRS $^{2}$ library provides the implementation of many tensor based decomposition methods for FG detection. The BG sequence is considered using the underlying multi-dimensional subspace called low-rank tensor and sparse error constitutes the moving FG objects. Fig. 1 shows an example of BS using tensor decomposition, whereas Fig. 2 indicates only frontal slice of tensor of original sequences taken from CDnet [18] and iLIDS [3] dataset for better visualisation.

However, HORPCA based decomposition methods into low-rank and sparse component currently face some prominent challenges: First, the state of the art HORPCA [7] based approaches are based on batch optimization processing. In order to perform decomposition, a number of training frames are required to store in a memory before processing any data. Therefore, due to the high memory storage, they are not applicable to real-time processing. Second, HORPCA based algorithms are sensitive to outliers, i.e., low-rank tensor recovery is not always robust, especially for BG/FG separation where outliers always appear and hence it is not applicable for practical visual surveillance systems. For example, the last row in Fig. 2 depicts an example of outlier appearances in frontal slice of the tensor. Third, earlier HORPCA approaches need to access every frame for Higher Order Singular Value Decomposition (HOSVD) as a result the computational problems prevent them from efficient processing of big data.

\footnotetext{
${ }^{1}$ https://github.com/andrewssobral/bgslibrary

${ }^{2} \mathrm{https} / / /$ github.com/andrewssobral//rslibrary
} 


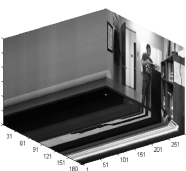

(a)

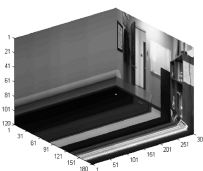

(b)

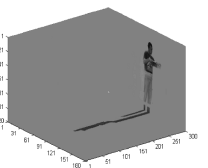

(c)

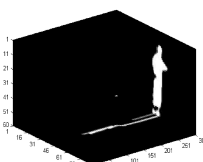

(d)
Figure 1. An example of input tensor decomposition: (a) input, (b) low-rank, (c) sparse tensor, and (d) foreground mask.

In order to address these major difficulties for BG/FG segmentation. This paper presents a robust recovery of low-rank tensor model for accurate FG segmentation. We briefly explain our methodology here. Traditional approaches are required to access each sample for HOSVD computation and therefore its nuclear norm tightly couples all the samples before processing. In this work, the stochastic (a.k.a online or iterative) optimization scheme where nuclear norm is re-formulated, recently proposed by Feng and $\mathrm{Xu}$ [6], is applied on each mode of the tensor. Our main technical contribution is an equivalent formulation of online RPCA [6] applied on tensor therefore we named it as, Online Tensor Decomposition (OTD) into low-rank and sparse component. OTD processes one sample per time instance from each vectorized tensor mode via online manners and hence reduces the memory usage and computational time. The low-rank tensor is then modeled by explicit product of basis and its coefficients, whereas the FG detection is obtained by thresholding the frontal slice of sparse tensor.

The rest of this paper is organized as follows. In Section 2, the related work is reviewed. Section 3 describes the proposed stochastic tensor decomposition for BS. Experimental results are discussed in Section 4, and finally Section 5 concludes our work.

\section{Previous Work}

In the literature, several algorithms have been proposed to cope with low-rank and sparse decomposition problem in computer vision. For example, Candes et al. [5] designed a very interesting framework called RPCA via Principal Component Pursuit (PCP) to decompose the given observation matrix into lowrank and sparse component. Excellent survey on BS using RPCA can be found in [2]. But these RPCA [5] matrix based decomposition methods used for BS [2] work only on single dimension and consider image as a vector and hence multidimensional data for efficient analysis can not be considered. In addition, the local spatial information sometimes lost and erroneous FG regions are obtained.

Therefore, tensor based algorithm called TSA (Tensor subspace learning) is developed by He et al. [8]. TSA is a very robust method for learning a subspace basis using multidimensional data but does not provide the convergence analysis. Wang et al. [17] proposed a convergent solution for tensor based low-rank learning. In addition, WTA (Window-based Tensor), STA (Streaming Tensor Analysis) and DTA (Dynamic Tensor Analysis) have been proposed by Sun et. al. [15]. In [11], RSTD (Robust Subspace Tensor Decomposition) is developed for automatic robust subspace recovery using Block

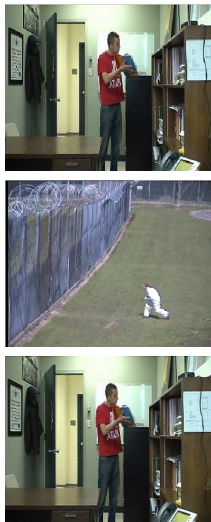

(a)
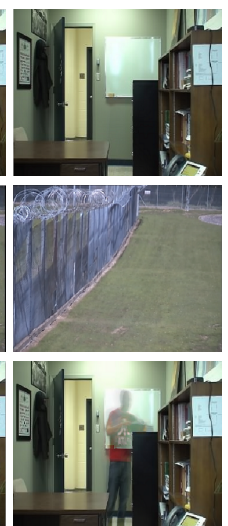

(b)

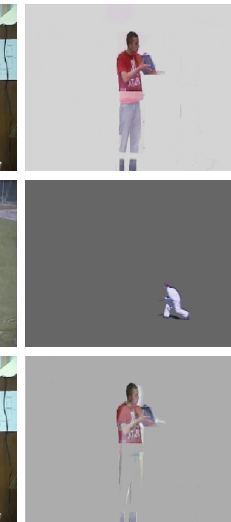

(c)
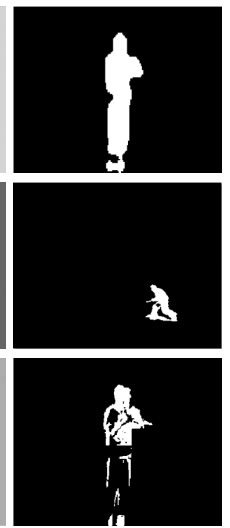

(d)
Figure 2. Frontal slice of tensor: (a) input, (b) low-rank, (c) sparse component, and (d) foreground mask.

Coordinate Descent (BCD) approach on unconstrained problem via variable splitting strategy, a number of computer vision applications such as image restoration, BS and face recognition are addressed in [11]. But parameters tuning and complexity of optimization method are the main drawbacks in RSTD. Donald and Qin [7] developed an extended Alternating Direction Augmented Lagrangian (ADAL) and HORPCA methods for robust tensor recovery. Convergence guarantee and proofs of each method are presented in [7]. Recently, Zhao et al. [19] proposed a Robust Bayesian Tensor Factorization (BRTF) scheme for incomplete tensor completion data. BRTF provides a very fast multi-way data convergence but tunning of annoying parameters and batch processing are the major difficulties in this approach.

All these matrix or tensor based decomposition methods discussed above are based on batch optimization mode. Therefore, a real time processing is loss due to the major challenges presented above. In order to tackle these problems, this paper presents a robust BS algorithm based on stochastic decomposition of low-rank and sparse component. We apply the idea of online optimization of RPCA [6] on tensor and update the individual basis iteratively followed by the processing of one frame per time instance using each unfolding matrices of tensor.

\section{Proposed Methodology}

In this section, stochastic tensor decomposition for robust BG/FG separation is presented. First, we describe some tensor basics and notations, then the proposed algorithm is presented in detail.

\subsection{Tensor Basics and Notation}

Tensor is considered as a multi-dimensional numerical array. The notations used in this paper are similar as used in [9]. The order of a tensor is the number of its dimensions (a.k.a modes or ways). The bold face calligraphic letters represent higher order tensors, (order $\geq 2$ ) e.g., $\mathcal{X}$. Scalars are represented by lowercase letter e.g., $\mathrm{x}$, whereas vectors and matrices are denoted by boldface lowercase and capital letters e.g., $\mathbf{x}$, and $\mathbf{X}$, 
respectively.

Let say an $N$ th order tensor $\mathcal{X} \in \mathbb{R}^{I_{1} \times I_{2} \times \ldots \times I_{N}}$, then its elements are denoted by symbolic name with indexes. For example, the element $(i, j, k)$ of third-order tensor $\mathcal{X}$ is represented by $x_{i, j, k}$. The analogue of matrix rows and columns for the tensors are called fibers, which is obtained by fixing all indexes but one (a.k.a vector-values subtensor). A tensor $\mathcal{X}$ has column, row and tube fibers represented by $x_{: j k}, x_{i: k}$, and $x_{i j:}$. Similarly, slices of a tensor are two dimensional sub-array that can be obtained by fixing all indexes but two. A tensor $\mathcal{X}$ has horizontal, lateral and frontal slices indicated by $x_{i::}, x_{j::}$, and $x_{k::}$. Reshaping is always required for manipulation of tensors, a common operation is called matrix unfolding or matricization for reformatting tensors into matrix. For $N$ th order tensor, its unfolding matrices are denoted by $\mathcal{X}_{1}, \mathcal{X}_{2}, \ldots, \mathcal{X}_{N}$. Vectorization of tensor into vector is denoted by $\operatorname{vec}(\mathcal{X})$. The squared Frobenius norm of a tensor is $\|\mathcal{X}\|_{F}^{2}=\langle\mathcal{X}, \mathcal{X}\rangle$. More details on tesor operations can be found in [9].

\subsection{Stochastic Tensor Decomposition}

Let say that $\mathcal{Y}$ is an input $N$ th order observation tensor, which is corrupted by outliers, say $\mathcal{E}$, then $\mathcal{Y}$ can be reconstructed by separating it into low-rank tensor $\mathcal{X}$ (corresponds to $\mathrm{BG}$ ), and sparse error $\mathcal{E}$ (corresponds to $\mathrm{FG}$ objects), i.e., $\mathcal{Y}=\mathcal{X}+\mathcal{E}$, under the convex optimization framework developed in [7] as

$$
\min _{\mathcal{X}, \mathcal{E}} \frac{1}{2} \sum_{i=1}^{N}\left\|\mathcal{Y}_{i}-\mathcal{X}_{i}-\mathcal{E}_{i}\right\|_{F}^{2}+\lambda_{1}\left\|\mathcal{X}_{i}\right\|_{*}+\lambda_{2}\left\|\mathcal{E}_{i}\right\|_{1},
$$

where $\left\|\mathcal{X}_{i}\right\|_{*}$ and $\left\|\mathcal{E}_{i}\right\|_{1}$ denote the nuclear and $l_{1}$ norm of each mode-i unfolding matrices of $\mathcal{X}$ and $\mathcal{E}$, respectively. Effcient methods such as CANDECOMP/PARAFAC (CP)decomposition and Tucker decompostion [9] (a.k.a HOSVD) are used for low-rank decomposition of tensor. In addition, APG, HORPCA-s based on ADAL and HORPCA-M based on I-ADAL are also developed in [7] to solve Eq.1. However, as mentioned above, these methods are based on batch optimization and not suitable for scalable data.

In this work, online optimization is considered to solve Eq.1. The major challenge is the computation of HOSVD because nuclear norm keeps all the samples tightly and therefore all samples are accessed during optimization at each iteration. Therefore, it suffers from high computational complexities. In constrast, an equivalent nuclear norm is used in this work for each mode-i unfolding matrices of $\mathcal{X}$, whose rank is upper bounded as shown in [13] as

$$
\begin{aligned}
&\left\|\mathcal{X}_{i}\right\|_{*}=\inf _{\mathbf{L} \in \mathbb{R}^{p \times r}, \mathbf{R} \in \mathbb{R}^{n \times r}}\left\{\frac{1}{2}\left(\left\|\mathbf{L}_{i}\right\|_{F}^{2}+\left\|\mathbf{R}_{i}\right\|_{F}^{2}\right)\right. \\
&\text { s.t. } \left.\mathcal{X}_{i}=\mathbf{L}_{\mathbf{i}} \mathbf{R}_{\mathbf{i}}^{T}\right\},
\end{aligned}
$$

where $p$ denotes the dimension of each sample e.g., width $\times$ height, $n$ is the number of samples and $r$ is a rank. Eq. 2 shows that mode-i unfolding matrices of low-rank tensor $\mathcal{X}$ can be an explicit product of each low-dimensional subspace basis $\mathbf{L} \in$
$\mathbb{R}^{p \times r}$ and its coefficients $\mathbf{R} \in \mathbb{R}^{n \times r}$ and this re-formulated nuclear norm is shown in recent works [4], [13] [14]. Hence, Eq. 1 is re-formulated by substituting Eq. 2 as

$$
\begin{aligned}
& \min _{\mathcal{X}_{1}, \ldots, \mathcal{X}_{N}, L} \in \mathbb{R}^{p \times r}, R \in \mathbb{R}^{n \times r}, \mathcal{E} \\
& \frac{1}{2} \sum_{i=1}^{N}\left\|\mathcal{Y}_{i}-\mathcal{X}_{i}-\mathcal{E}_{i}\right\|_{F}^{2}+ \\
& \frac{\lambda_{1}}{2}\left(\left\|\mathbf{L}_{i}\right\|_{F}^{2}+\left\|\mathbf{R}_{i}\right\|_{F}^{2}\right)+\lambda_{2}\|\mathcal{E}\|_{1} \text {, s.t. } \mathcal{X}_{i}=\mathbf{L}_{\mathbf{i}} \mathbf{R}_{\mathbf{i}}{ }^{T} .
\end{aligned}
$$

For objective function minimization, avoiding the constraints in Eq.3 and put $\mathcal{X}_{i}=\mathbf{L}_{\mathbf{i}} \mathbf{R}_{\mathbf{i}}{ }^{T}$ as

$$
\begin{array}{r}
\min _{\mathcal{X}_{1}, \ldots, \mathcal{X}_{N}, L \in \mathbb{R}^{p \times r}, R \in \mathbb{R}^{n \times r}, \mathcal{E}} \frac{1}{2} \sum_{i=1}^{N}\left\|\mathcal{Y}_{i}-\mathbf{L}_{i} \mathbf{R}_{i}^{T}-\mathcal{E}_{i}\right\|_{F}^{2}+ \\
\frac{\lambda_{1}}{2}\left(\left\|\mathbf{L}_{i}\right\|_{F}^{2}+\left\|\mathbf{R}_{i}\right\|_{F}^{2}\right)+\lambda_{2}\|\mathcal{E}\|_{1},
\end{array}
$$

where $\lambda_{1}$ and $\lambda_{2}$ are regularization parameters for low-rank and sparsity patterns. E.q 5 is the main equation for stochastic tensor decomposition which is not completely convex with respect to $\mathbf{L}$ and $\mathbf{R}$. However, E.q. 3 is the global optimal solutions to the original optimization problem in E.q. 2 as proved in [6]. The following cost function is required to optimize for solving E.q 3 as

$$
f_{n}(\mathbf{L})=\frac{1}{n} \sum_{i=1}^{N} \sum_{t=1}^{n} l\left(\mathcal{Y}_{i}^{t}, \mathbf{L}_{i}\right)+\frac{\lambda_{1}}{2 n}\left\|\mathbf{L}_{i}\right\|_{F}^{2},
$$

where $\mathcal{Y}_{i}^{t}$ denotes $i^{\text {th }}$ mode of a tensor $\mathcal{Y}$ at a time $t$ instance given by

$$
l\left(\mathcal{Y}_{i}^{t}, \mathbf{L}\right)=\min _{\mathbf{r}, \mathbf{e}}\left\|\operatorname{vec}\left(\mathcal{Y}_{i}^{t}\right)-\mathbf{L r}-\mathbf{e}\right\|_{2}^{2}+\frac{\lambda_{1}}{2}\|\mathbf{r}\|_{2}^{2}+\lambda_{2}\|\mathbf{e}\|_{1}
$$

Finally, the objective function $l_{t}(\mathbf{L})$ for updating the modei basis $\mathbf{L}_{i}$ matrix of multidimensional subspace tensor $\mathcal{X}$ at a time $t$ instance is given by

$$
\begin{aligned}
l_{t}\left(\mathbf{L}_{i}\right)=\frac{1}{n} \sum_{t=1}^{n} & \left\{\frac{1}{2}\left\|\operatorname{vec}\left(\mathcal{Y}_{i}^{t}\right)-\mathbf{L}_{i}^{t} \mathbf{r}^{t}-\mathbf{e}^{t}\right\|_{2}^{2}+\frac{\lambda_{1}}{2}\left\|\mathbf{r}^{t}\right\|_{2}^{2}\right. \\
& \left.+\lambda_{2}\left\|\mathbf{e}^{t}\right\|_{1}\right\}+\frac{\lambda_{1}}{2 t}\left\|\mathbf{L}_{i}^{t}\right\|_{F}^{2},
\end{aligned}
$$

where $\mathbf{r}^{t}$ and $\mathbf{e}^{t}$ are vectors of coefficient and noise at a time $t$ for matrix $R_{i}$, respectively, and mode-i matrix of sparse tensor $\mathcal{E}$. The main goal is to minimize the cost function E.q. 6 through stochastic optimization method as shown in Algorithm 1.

In case of BG modeling, one video frame at a time $t$ is processed in an online manners. The coefficient $\mathbf{r}$, sparse matrix $\mathbf{e}$ and basis $\mathbf{L}$ are optimized in an iterative way. First, the coefficient $\mathbf{r}$ and noise e matrices are estimated with fixed random basis $\mathbf{L}$ by projecting one sample using Eq. 11. This subproplem in step. 6 requires to solve a following small-scale convex optimization problem at a time instance $t$ as 


$$
\begin{gathered}
\mathbf{r}^{t}=\left(\mathbf{L}^{T} \mathbf{L}+\lambda_{1} \mathbf{I}\right)^{-1} \mathbf{L}^{T}\left\{\operatorname{vec}\left(\mathcal{Y}_{i}^{t}\right)-\mathbf{e}^{t-1}\right\}, \\
e^{t}= \begin{cases}\mathbf{M}^{t}(k)-\lambda_{2}, & \text { if } \mathbf{M}^{t}(k)>\lambda_{2}, \\
\mathbf{M}^{t}(k)+\lambda_{2}, & \text { if } \mathbf{M}^{t}(k)<\lambda_{2}, \\
0, & \text { otherwise, }\end{cases}
\end{gathered}
$$

where $\mathbf{M}=\operatorname{vec}\left(\mathcal{Y}_{i}^{t}\right)-\mathbf{L} \mathbf{r}^{t}$ and $\mathbf{M}_{t}(k)$ is the $k^{t h}$ element in $\mathbf{M}$ at a time $t$. Second, the basis $\mathbf{L}^{t}$ is estimated using the E.q. 14 through minimizing the previously computed coefficients $\mathbf{r}$ and $\mathbf{e}$. These basis $\mathbf{L}^{t}$ for low-dimensional subspace learning is then updated using Algorithm 2 by the results of $\mathbf{r}$ and e. If the rank $r$ is given and basis $L$ are estimated as above which is a fully rank, then $L$ converges to the optimal solution asymptotically as compared to its batch counterpart as shown in [6].

Finally each $i^{\text {th }}$ mode low-dimensional subspace tensor $\mathcal{X}$ is estimated by a multiple of basis $\mathbf{L}$ and coefficients $\mathbf{R}$. The BG sequence is then modeled by low-rank tensor $\mathcal{X}$ which changes at a time instance $t$, whereas the resulting sparse tensor $\mathcal{E}$ is obtained by the matricization of e entries. Finally, a hard thresholding scheme is applied on a sparse component to get the binary FG mask for BG/FG separation.

\section{Experimental Evaluations}

In this section, we present our experimental results in detail. We first evaluate the proposed algorithm performance on synthetic data then the qualitative and quantitative analysis using Change Detection (CDnet) [18] and Background Models Challenge (BMC) [16] dataset for real time BG/FG segmentation is presented in detail.

\subsection{Evaluation on Synthetic Data}

The proposed method is first quantitatively tested on synthetic data. For data evaluation, a true low-rank tensor $\mathcal{X}$ of size $30 \times 30 \times 30$ is generated by rank-3 factor matrices e.g., $\mathbf{Z}^{n} \in \mathbb{R}^{30 \times 3}$ where $n=1,2,3$. Each factor matrix $\mathbf{Z}^{n}$ consists of three components such as $\left[\sin \left(\frac{2 \pi n i_{n}}{30}\right), \cos \left(\frac{2 \pi n i_{n}}{30}\right), \operatorname{sgn}\left(\sin \left(0.5 \pi i_{n}\right)\right)\right]$. The first two components are different and third one is common in all modes. A random entries of this low-rank tensor $\mathcal{X}$ is corrupted by outliers from uniform distribution $U(-|H|,|H|)$ and small noise $N(0,0.01)$ is also considered. We use a well known measure for evaluation called "Root Relative Square Error" (RRSE) given by $\frac{\|\widehat{\mathcal{X}}-\mathcal{X}\|_{2}}{\|\mathcal{X}\|_{2}}$, where $\widehat{\mathcal{X}}$ is a reconstructed low-rank tensor. We compare our RRSE performance with earlier methods based on batch optimization processing such as BRTF [19], CP-ARD [12], CP-ALS [9], HORPCA [7], and HOSVD [7], respectively. Fig. 3 shows the value of RRSE with results of reconstructed tensor $\widehat{\mathcal{Y}}$. We consider two cases for robust tensor recovery for true data generation in Fig. 3. First, the magnitude is considered within a range of true data as shown in Fig. 3 (a). However, Fig. 3 (b) shows that the magnitude is taken larger

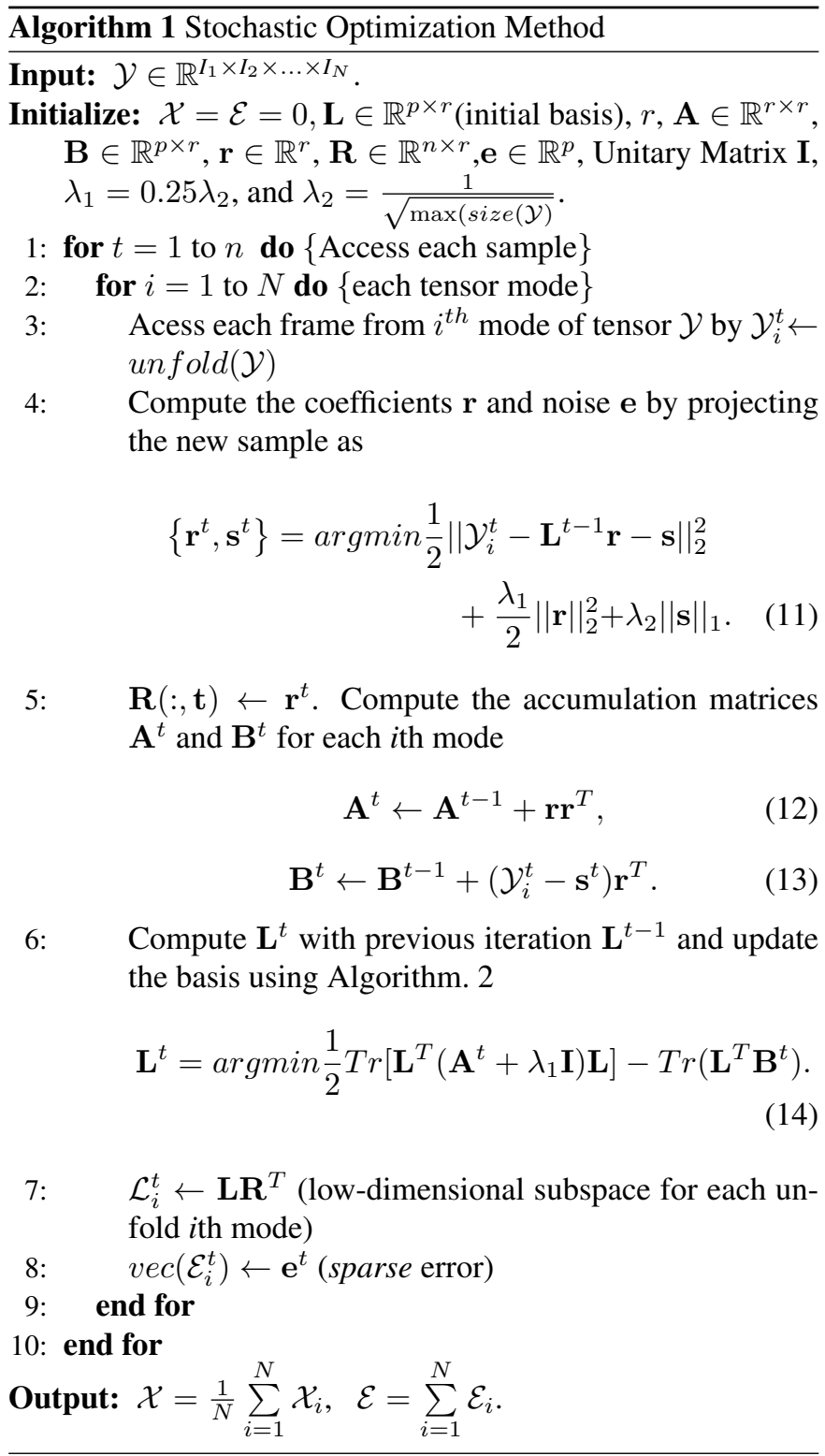

for corrupting some entries in true low-rank. In each case, the proposed method outperforms with previous HORPCA methods. However, it shows a comparative performance to its batch counter-part such as BRTF. The proposed scheme processes one frame per time instance and achieve almost a real-time processing, whereas BRTF is based on batch optimization strategy and hence it is not applicable for processing high dimensional data.

\subsection{Evaluation on CDnet and BMC Dataset for BS}

We have also evaluated our method for robust BS using synthetic and real videos of BMC and CDnet dataset. Due to the space limitations, we only present 5 selected synthetic videos of BMC dataset for qualitative analysis. The image size of each video sequence is $640 \times 480$ and half of the resolution is used. Both grayscale and color videos are evaluated. The size of grayscale input tensor $\mathcal{Y}$ is $320 \times 240 \times 200$ with 200 frames 

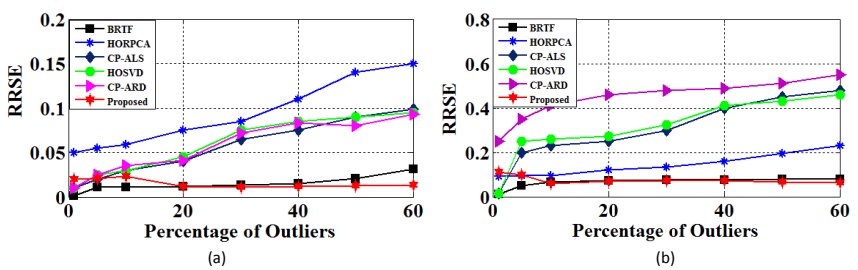

Figure 3. Performance of reconstructed low-rank tensor, (a) $\mathrm{O}=\max (\operatorname{vec}(\mathcal{X}))$, and (b) $\mathrm{O}=10 \cdot \operatorname{std}(\operatorname{vec}(\mathcal{X}))$.

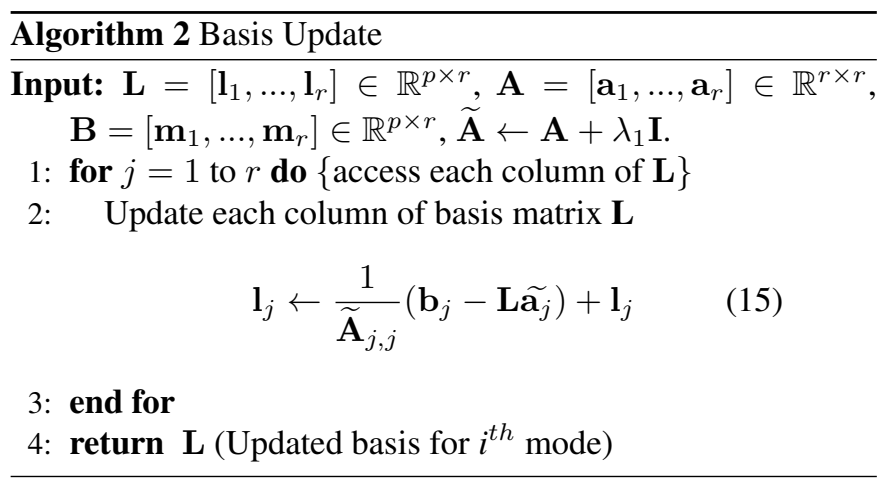

and color video frame tensor as $320 \times 240 \times 3$. Stochastic tensor decomposition is applied on each video frame to separate $\mathcal{X}$ and $\mathcal{E}$ component. The value of rank $r=10$ in Eq. 5 above. Additionally, median filtering is applied as a post-processing step to filter binary mask for morphological smoothing. Fig. 4 (i)-(m) shows the visual results of synthetic sequences from Street and Rotary category of BMC dataset [16].

From CDnet dataset [18], 8 real videos from category Baseline and Thermal are tested to validate the proposed scheme. The resolution of each sequence is $320 \times 240 \times 3$ and only color videos are tested. So the size of input tensor $\mathcal{Y}$ is $320 \times 240 \times 3 \times n$, where $n$ is the number of input video frames and it varies from 500 to 4,000 according to the sequences presented in CDnet dataset. The Thermal category contains real videos under increasing percentage of practical camera noise. Fig. 4 (a)-(h) depicts the visual results on 8 real videos selected from Baseline and Thermal category.

The proposed scheme is also evaluated for quantitative analysis. A well known $F$ - measure metric is computed as $F=\frac{2 \times \text { Recall } \times \text { Precision }}{\text { Recall }+ \text { Precision }}$ by comparing our results with available corresponding ground truth images. Table 1 shows that our proposed method is the top performer as compared to earlier batch optimization based algorithms. The "-" line in table 1 means that these methods are not applicable to large scale videos $(n>500)$ due to the batch optimization methods. Computational complexity is also observed during our experiments. Time is recorded in CPU time as $[h h: m m: s s]$ and table 2 shows the computational time of each method for first 100 frames with varying image resolution. All the experiments are conducted in Matlab R2013a with $3.40 \mathrm{GHz}$ Intel core i5 processor with 4 GB RAM. These all robust experimental evaluations are the consequences of our proposed scheme.

\begin{tabular}{|c|c|c|c|c|}
\hline Method & HORPCA & CP-ALS & BRTF & OTD \\
\hline $160 \times 120$ & $00: 01: 35$ & $00: 00: 40$ & $00: 00: 22$ & $\mathbf{0 0 : 0 0 : 0 4}$ \\
\hline $320 \times 240$ & $00: 04: 56$ & $00: 02: 09$ & $00: 03: 50$ & $\mathbf{0 0 : 0 0 : 1 2}$ \\
\hline
\end{tabular}

Table 2. Computational time according to different image resolutions.

\section{Conclusion}

In this paper, we proposed stochastic tensor decomposition for robust BS application. Experimental results show that we have achieved almost a real time processing since one frame is processed according to online optimization scheme. The coefficients and noise matrix are obtained using randomized basis then the basis is updated which is very robust against gross outliers. However, we have not integrated any robust features neither we applied this idea on highly dynamic BG scenes. Therefore, we will extend this work by fusing robust features such as disparity and texture information for more robust FG detection. Moreover, this idea can be used for other computer vision tasks such as online tracking using low-rank sparse tensor as a tracker.

\section{Acknowledgement}

This work is supported by the World Class 300 project, Development of HD video/network-based video surveillance system(10040370), funded by the Ministry of Trade, Industry, and Energy (MOTIE, Korea).

\section{References}

[1] Thierry Bouwmans, Fida El Baf, Bertrand Vachon, et al. Statistical Background Modeling for foreground detection: A survey. Handbook of Pattern Recognition and Computer Vision, pages 181-199, 2010.

[2] Thierry Bouwmans and El Hadi Zahzah. Robust PCA via Principal Component Pursuit: A review for a comparative evaluation in video surveillance. Computer Vision and Image Understanding, pages 22-34, 2014.

[3] Home Office Scientific Development Branch. Imagery library for intelligent detection systems I-LIDS. In Crime and Security, 2006. The Institution of Engineering and Technology Conference on, pages 445-448, June 2006.

[4] Samuel Burer and Renato DC Monteiro. A nonlinear programming algorithm for solving semidefinite programs via low-rank factorization. Mathematical Programming, 95(2):329-357, 2003.

[5] Emmanuel J Candès, Xiaodong Li, Yi Ma, and John Wright. Robust Principal Component Analysis? Journal of the ACM (JACM), 58(3):11-37, 2011.

[6] Jiashi Feng, Huan Xu, and Shuicheng Yan. Online Robust PCA via Stochastic Optimization. In Advances in Neural Information Processing Systems, pages 404-412, 2013. 


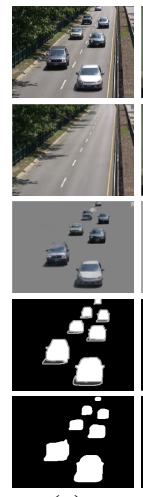

(a)

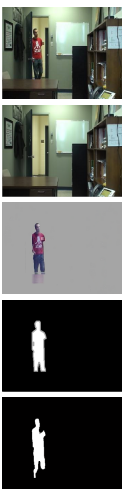

(b)

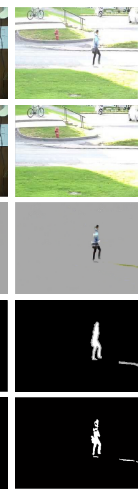

(c)

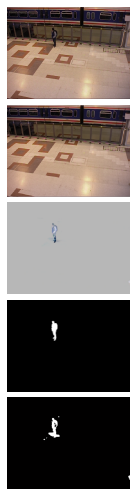

(d)

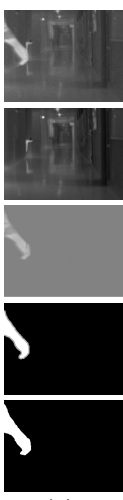

(e)

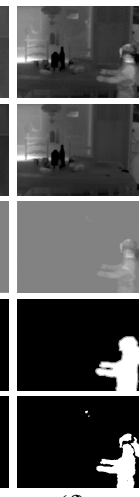

(f)

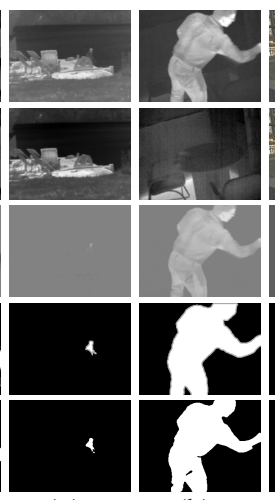

(g)

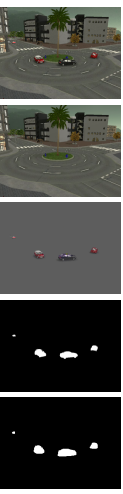

(i)

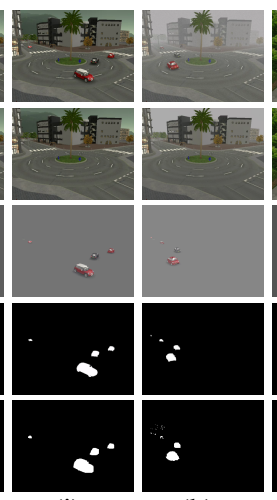

(k)

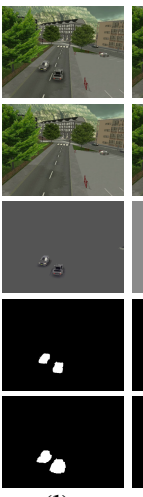

(1)

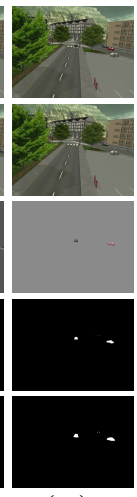

(m)

4. Qualitative results of CDnet [18] and BMC [16] dataset. From (a) to (d) Baseline, and (e) to (h) Thermal category of CDnet, and (i)-(m) Synthetic videos of BMC. From top to bottom, input, low-rank, sparse, ground truth, and binary mask of proposed methodology.

Table 1. Qualitative results of BMC [16] and CDnet [18] dataset: Average F-measure score in (\%) on each video.

\begin{tabular}{|c|c|c|c|c|c|c|c|c|c|c|c|c|c|c|c|c|c|c|c|c|}
\hline \multirow[t]{3}{*}{ Method } & \multicolumn{10}{|c|}{ BMC [16] } & Average & \multicolumn{8}{|c|}{ CDnet [18] } & \multirow[t]{3}{*}{ Average } \\
\hline & \multicolumn{5}{|c|}{ Street } & \multicolumn{5}{|c|}{ Rotary } & & \multicolumn{4}{|c|}{ Baseline } & \multicolumn{4}{|c|}{ Thermal } & \\
\hline & 112 & 212 & 312 & 412 & 512 & 122 & 222 & 322 & 422 & 522 & & highway & office & pedestrians & PETS2006 & corridor & diningRoom & lakeSide & library & \\
\hline HORPCA [7] & 81.30 & 84.33 & 69.94 & 69.46 & 75.63 & 82.34 & 86.10 & 72.45 & 67.20 & 72.56 & 76.13 & 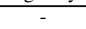 & 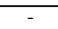 & - & - & - & - & - & 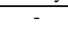 & - \\
\hline CP-ALS [9] & 84.70 & 84.21 & 78.65 & 76.32 & 79.12 & 85.36 & 81.23 & 71.60 & 78.05 & 80.52 & 79.97 & - & - & - & - & - & - & - & - & - \\
\hline BRTF [19] & 87.83 & 85.63 & 81.10 & 82.29 & 84.13 & \begin{tabular}{|l|}
88.10 \\
\end{tabular} & 90.36 & 85.55 & 80.69 & 86.61 & 85.22 & - & - & - & - & - & - & - & - & - \\
\hline Ours & 91.65 & 87.10 & 84.80 & 78.61 & 85.93 & 93.35 & 90.21 & 87.65 & 81.20 & 84.63 & 86.50 & 88.36 & 85.92 & 90.63 & 74.41 & 86.30 & 80.68 & 76.58 & 94.66 & 84.19 \\
\hline
\end{tabular}

[7] Donald Goldfarb and Zhiwei Qin. Robust low-rank tensor recovery: Models and algorithms. SIAM Journal on Matrix Analysis and Applications, 35(1):225-253, 2014.

[8] Xiaofei He, Deng Cai, and Partha Niyogi. Tensor subspace analysis. In Advances in neural information processing systems, pages 499-506, 2005.

[9] Tamara G Kolda and Brett W Bader. Tensor decompositions and applications. SIAM review, 51(3):455-500, 2009.

[10] Wang Hee Lee, Kuntal Sengupta, and Rajeev Sharma. Augmented reality with occlusion rendering using background-foreground segmentation and trifocal tensors. In Multimedia and Expo, 2003. ICME'03. Proceedings. 2003 International Conference on, volume 2, pages II-93. IEEE, 2003.

[11] Yin Li, Junchi Yan, Yue Zhou, and Jie Yang. Optimum subspace learning and error correction for tensors. In Computer Vision-ECCV 2010, pages 790-803. Springer, 2010 .

[12] Morten Mørup and Lars Kai Hansen. Automatic relevance determination for multi-way models. Journal of Chemometrics, 23(7-8):352-363, 2009.

[13] Benjamin Recht, Maryam Fazel, and Pablo A Parrilo. Guaranteed minimum-rank solutions of linear matrix equations via nuclear norm minimization. SIAM review, 52(3):471-501, 2010.
[14] Jasson DM Rennie and Nathan Srebro. Fast maximum margin matrix factorization for collaborative prediction. In Proceedings of the 22nd international conference on Machine learning, pages 713-719. ACM, 2005.

[15] Jimeng Sun, Dacheng Tao, Spiros Papadimitriou, Philip S $\mathrm{Yu}$, and Christos Faloutsos. Incremental tensor analysis: Theory and applications. ACM Transactions on Knowledge Discovery from Data (TKDD), 2(3):11, 2008.

[16] Antoine Vacavant, Thierry Chateau, Alexis Wilhelm, and Laurent Lequièvre. A Benchmark Dataset for Outdoor Foreground/Background Extraction. In Computer VisionACCV 2012 Workshops, pages 291-300. Springer, 2013.

[17] Huan Wang, Shuicheng Yan, Thomas S Huang, and Xiaoou Tang. A convengent solution to tensor subspace learning. In IJCAI, pages 629-634, 2007.

[18] Yi Wang, Pierre-Marc Jodoin, Fatih Porikli, Janusz Konrad, Yannick Benezeth, and Prakash Ishwar. Cdnet 2014: An expanded change detection benchmark dataset. In Computer Vision and Pattern Recognition Workshops (CVPRW), 2014 IEEE Conference on, pages 393-400. IEEE, 2014.

[19] Qibin Zhao, Guoxu Zhou, Liqing Zhang, Andrzej Cichocki, and Shun-ichi Amari. Robust Bayesian Tensor Factorization for Incomplete Multiway data. arXiv preprint arXiv:1410.2386, 2014. 